\title{
LOW MACH NUMBER LIMIT OF MULTIDIMENSIONAL STEADY FLOWS ON THE AIRFOIL PROBLEM
}

\author{
MINGJIE LI, TIAN-YI WANG, AND WEI XIANG
}

\begin{abstract}
In this paper, we justify the low Mach number limit of the steady irrotational Euler flows for the airfoil problem, which is the first result for the low Mach number limit of the steady Euler flows in an exterior domain. The uniform estimates on the compressibility parameter $\varepsilon$, which is singular for the flows, are established via a variational approach based on the compressible-incompressible difference functions. The limit is on the Hölder space and is unique. Moreover, the convergence rate is of order $\varepsilon^{2}$. It is noticeable that, due to the feature of the airfoil problem, the extra force dominates the asymptotic decay rate of the compressible flow to the infinity. And the effect of extra force vanishes in the limiting process from compressible flows to the incompressible ones, as the Mach number goes to zero.
\end{abstract}

\section{INTRODUCTION}

We are concerned with the low Mach number limit of steady homentropic Euler flows to the airfoil problem. The steady homentropic Euler equations with the extra force are written as:

$$
\left\{\begin{array}{l}
\operatorname{div}(\rho u)=0, \\
\operatorname{div}(\rho u \otimes u)+\nabla p=\rho F,
\end{array}\right.
$$

where $x=\left(x_{1}, \cdots, x_{n}\right) \in \mathbb{R}^{n}, n \geq 3, u=\left(u_{1}, \cdots, u_{n}\right)$ is the velocity, $\rho, p$ and $F$ represent the density, pressure, and extra forces respectively. Moreover, $u \otimes u=\left(u_{i} u_{j}\right)_{n \times n}$ is an $n \times n$ matrix. Through this paper, we consider that the extra force $F$ is conservative. This is reasonable since this type of forces is quite natural and important in the reality. For instance, the gravity field is a conservative field. Another important example is the electric field. As the homentropic flow, the pressure is a function of density as:

$$
p:=\frac{\tilde{p}(\rho)-\tilde{p}(1)}{\varepsilon^{2}},
$$

where $\varepsilon>0$ is the compressibility parameter as introduced in [34]. As usual, we require

$$
\tilde{p}^{\prime}(\rho)>0, \quad 2 \tilde{p}^{\prime}(\rho)+\rho \tilde{p}^{\prime \prime}(\rho)>0 \quad \text { for } \rho>0 .
$$

We remark that condition $(1.3)$ holds for the flows governed by the thermodynamic relation that $\tilde{p}=\rho^{\gamma}$ with $\gamma \geq 1$. The sound speed of the flow is

$$
c:=\sqrt{p^{\prime}(\rho)}=\frac{\sqrt{\tilde{p}^{\prime}(\rho)}}{\varepsilon},
$$

Date: March 22, 2022.

2010 Mathematics Subject Classification. 35Q31; 35L65; 76N15; 35B40;

Key words and phrases. Multidimensional, low Mach number limit, steady flow, homentropic Euler equations, convergence rate. 
and the Mach number is defined as

$$
M:=\frac{|u|}{c}=\frac{\varepsilon|u|}{\sqrt{\tilde{p}^{\prime}(\rho)}} .
$$

Formally, if $|u|$ is bounded and $\sqrt{\tilde{p}^{\prime}(\rho)}$ does not vanish, the Mach number will go to 0 as $\varepsilon \rightarrow 0$. For this reason, the limit $\varepsilon \rightarrow 0$ is called the low Mach number limit.

The expected corresponding homogeneous incompressible Euler equations as $\varepsilon \rightarrow 0$ are written as:

$$
\left\{\begin{array}{l}
\operatorname{div} \bar{u}=0, \\
\operatorname{div}(\bar{u} \otimes \bar{u})+\nabla \bar{p}=F,
\end{array}\right.
$$

where $\bar{u}=\left(\bar{u}_{1}, \cdots, \bar{u}_{n}\right)$ and $\bar{p}$ represents the velocity and pressure, respectively, while density $\bar{\rho} \equiv 1$.

To study the low Mach number limit, we should start from the case with sufficiently small Mach number. The flow is subsonic when the Mach number $M<1$. Since the equations corresponding to the mixed-type characteristics in the compressible Euler equations are elliptic for the subsonic flows, we can expect nice regularity compared to those related to the transonic flows or supersonic flows.

The airfoil problems studies the flow past through an exterior domain with the slip boundary condition, which equals to consider the flows around uniform motion body like the airfoil or car after the Galilean transformation. Both the compressible case and incompressible case have been studied by many authors. The first result is due to Frankl and Keldysh [15], who studied the subsonic flows around a two dimensional airfoil and proved the existence and uniqueness for small data by the method of successive approximations. By the variational method, Shiffman [35] proved that, if the speed of the flow at the infinity, $u_{\infty}$, is less than some critical speed, then there exists a unique subsonic potential flow around a given profile with finite energy. Shortly afterwards, Bers [2] improved the uniqueness results of Shiffman. Finn and Gilbarg [12] proved the uniqueness of the two dimensional potential subsonic flow about a bounded obstacle with given circulation and velocity at the infinity. All the above results are related to the two dimensional case. For the three (or higher) dimensional case, Finn and Gilbarg [13] proved the existence, uniqueness and the asymptotic behavior with implicit restrictions on the Mach number. Payne and Weinberger [31] improved the results soon after. Later, Dong and $\mathrm{Ou}$ [8] extended the results of Finn and Gilbarg [13] to any Mach number $M<1$ and to arbitrary dimensions by the direct method of the calculus of variations and the standard Hilbert space method. Recently, the result has been extended to the case with conservative force in [18]. The corresponding incompressible case is considered by $\mathrm{Ou}$ in [26, 30]. On the other hand, recently, there are many literitures on the steady compressible Euler equations (see [5, 7, 11, 17, 32, 37] for examples). So it is time for us to consider the low Mach number limit of steady flows.

It is well-known in physics that the compressible flow is expected to perform like an incompressible flow, when the Mach number is sufficiently small. However, how to rigorously justify the physical observation of this limit is a challenging mathematical problem, since it is a singular limit and singular phenomena are expected in the limit process. The first theory of the low Mach number limit is due to Janzen and Rayleigh (see [33, Sect. 47], 38]), which concerned with the steady irrotational flow. Their method of the expansion of solutions in power with respect to the Mach number was used both 
as a computational tool and as a method for the proof of the existence of solutions of the compressible flow. Klainerman and Majda [22, 23] proved the convergence of compressible to incompressible flow by directly obtaining estimates for the scaled form of the partial differential equations (also see Ebin [9]). In particular, under the suitable initial data, they showed the convergence rates of both Euler equations and Navier-Stokes equations are $\varepsilon$ order on velocity and $\varepsilon^{2}$ on density and pressure. By using the fast decay of acoustic waves, Ukai in [36] verified the low Mach number limit for the general data, while the exterior domain cases were considered in [19]. The major breakthrough on the general initial data is due to Métivier and Schochet [29], which is extended to the exterior domain problem by Alazard [1]. For the one dimensional case, under the $B V$ space, the low Mach number limit have been considered [3]. For the steady Euler flow, LiWang-Xiang [24] considered the infinitely long nozzle problem, which is the first rigorous analysis for the steady Euler equation. For other fluid models, see [14, 20, 21, 25, 27, 29] and the references therein. For another type of incompressible limit, please see [4, 28] and reference therein.

All the results mentioned above are related to the case that the boundary of the profile is smooth. For the case that the profile is a polygon, recently, Elling [10] showed that when the Mach number is sufficiently small but nonzero, the classical solution of the irrotational steady Euler flows around a polygon does not exist.

In this paper, we prove the limit is convergent strongly in the Hölder norm and is unique. Moreover, the convergence rate of order $\varepsilon^{2}$ is justified, which is higher than the one in Klainerman-Majda [22], due to the irrotational property. More precisely, the main difficulties for the steady Euler flow are how to get the uniform estimates on the velocity. Therefore, we introduce the variational functional with respect to the compressibleincompressible difference function to obtain the uniform $L^{2}$ estimates. Moreover, not only are the incompressible and compressible velocities the minimizer of the respective functional but also the compressible-incompressible difference velocity is the minimizer of a functional. The effect of the extra force comes out in the regularity lifting to the Hölder space naturally. In addtion, the effect dominates the asymptotic rate to the infinity for the compressible flows, but the incompressible flows are free from this effect. The vanishing phenomenon is rigorously justified by the proper expansion based on a suitable formulation on the Bernoulli's law and a refined cut-off function for the low Mach number limits. Another difficulty is to find a proper way to show the convergence of the pressure. Based on the observation that the pressure of the incompressible flow is well-defined up to a constant by the Bernoulli's law, we establish the convergence on the gradient of pressure.

The rest of this paper is organized as follows. In Section 2, we formulate the low Mach number limit of the steady irrotational Euler flows corresponding to the airfoil problem mathematically, and then introduce the main theorem of this paper. In Sections 3, we derive a variational formulation to show the existence and uniqueness of a minimizer of the variational problem. In Section 4, we show the minimizer is actually the unique solution of the compressible subsonic Euler flow in $\mathbb{R}^{n}$ of the airfoil problem with $C^{\alpha_{-}}$ regularity. Finally, the convergence rate of the low Mach number limit of the steady irrotational Euler flows is established. 

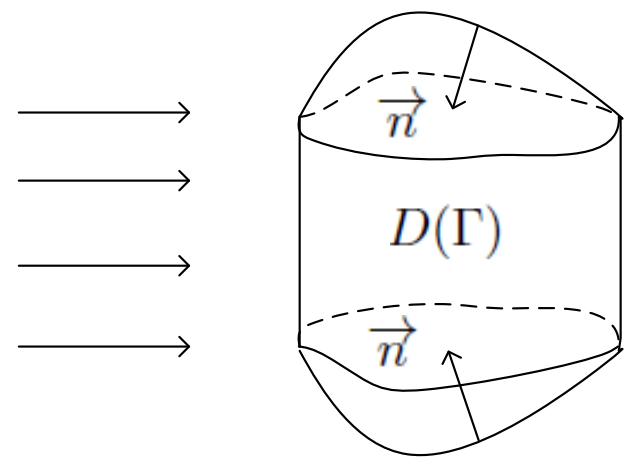

$\Omega$

Figure 2.1. Airfoil Problem

\section{Airfoil Problem and the Low Mach number Limit}

In this section, we will first introduce both the incompressible and compressible airfoil problem, then the low Mach number limit, and finally the main theorem of this paper.

2.1. Airfoil problem. Let $\mathcal{D}(\Gamma)$ (airfoil) be a bounded and connected domain in $\mathbb{R}^{n}$ $(n \geq 3)$ such that its boundary $\Gamma$ consists of one or several closed and isolated $n-1$ dimensional $C^{2, \alpha}$ (for some $0 \leq \alpha \leq 1$ ) hypersurfaces. Let $\Omega$ be the exterior domain of $\mathcal{D}(\Gamma)$, i.e., $\Omega:=\mathbb{R}^{n} \backslash \mathcal{D}$, which is connected (see Fig 2.1). Then both the incompressible and compressible airfoil problem can be formulated as the following problem.

Problem 1. Let $n \geq 3$. Find functions $(\rho, u, p)$ satisfy 1.1$)$ or functions $(u, p)$ satisfy (1.4) with the slip boundary condition

$$
u \cdot \vec{n}=0 \text { on } \Gamma
$$

where $\vec{n}=\left(n_{1}, \cdots, n_{n}\right)$ denotes the unit inward normal of domain $\mathcal{D}(\Gamma)$. Moreover, the limits

$$
\lim _{|x| \rightarrow \infty} u(x)=u_{\infty}
$$

and

$$
\lim _{|x| \rightarrow \infty} \rho(x)=1
$$

exist and are finite.

Remark 2.1. By the Galilean invariance of the Newton fluid, $\mathcal{D}$ can be assumed to be stationary. That is the reason that we assign boundary condition (2.1) on $\Gamma$. Without loss of the generality, we also assume that $u_{\infty}=\left(q_{\infty}, 0, \cdots, 0\right)$.

Since the extra force is conservative form, and velocity is asymptotic irrotational at infinite, we assume the flow is irrotational, which means the vorticity of the flow velocity is zero, i.e.,

$$
\operatorname{curl} u=0
$$

2.2. Incompressible airfoil problem. Now let us consider the existence of the incompressible flow for the airfoil problem. Due to [8, 26, 30], the suitable function space is of the following. 
LOW MACH NUMBER LIMIT OF MULTIDIMENSIONAL STEADY FLOWS ON THE AIRFOIL PROBLEN

Definition 2.1. $\mathcal{V}$ is the Hilbert space for $n \geq 3$, which is the completing of space $\mathcal{V}_{0}$ under the norm

$$
\|v\|_{\mathcal{V}}=\left(\int_{\Omega}|\nabla v|^{2} d x\right)^{\frac{1}{2}}
$$

Here $\mathcal{V}_{0}$ is the set of all the functions which are the restrictions of the $C_{c}^{\infty}\left(\mathbb{R}^{n}\right)$ functions on $\Omega$ :

$$
\mathcal{V}_{0}=\left\{v(x) \mid v(x)=V(x), x \in \Omega, \text { for some } V \in C_{c}^{\infty}\left(\mathbb{R}^{n}\right)\right\} .
$$

We remark that for the $C_{c}^{\infty}$ functions, $L^{2}$ norm of the gradient can be bounded by the norm $\|\cdot\|_{\mathcal{V}}$ due to the following Hardy type inequality.

Theorem 2.1. There exists a constant $c(n, \Omega)$ such that for any $v \in \mathcal{V}$

$$
\int_{\Omega} \frac{v^{2}}{1+|x|^{2}} d x \leq c(n, \Omega) \int_{\Omega}|\nabla v|^{2} d x, \quad \text { for } \quad n \geq 3 .
$$

Note that the steady irrotational incompressible Euler flow is governed by the following equations:

$$
\left\{\begin{array}{l}
\operatorname{div} \bar{u}=0, \\
\operatorname{div}(\bar{u} \otimes \bar{u})+\nabla \bar{p}=F, \\
\operatorname{curl} \bar{u}=0 .
\end{array}\right.
$$

Here, the density $\bar{\rho} \equiv 1$, and conservative force $F$ could be written as $F=\nabla \phi$. By $2.6{ }_{3}$, we can introduce the velocity potential $\bar{\varphi}$ such that

$$
\bar{u}=\nabla \bar{\varphi} .
$$

Therefore, the incompressible irrotational Euler flows of Problem 1 can be found by looking for solution $\bar{\varphi}$, which satisfies

$$
\begin{cases}\Delta \bar{\varphi}=0, & x \in \Omega, \\ \frac{\partial \bar{\varphi}}{\partial \mathbf{n}}=0, & x \in \Gamma, \\ \lim _{|x| \rightarrow \infty} \nabla \bar{\varphi}=\left(q_{\infty}, 0, \cdots, 0\right), & \end{cases}
$$

Due to the asymptotic behavior of $\nabla \bar{\varphi}$ at infinity (see $\left.(2.8)_{3}\right)$, we can not expect the $L^{2}$ estimate of $\nabla \bar{\varphi}$. So we introduce $\bar{\psi}$, such that

$$
\bar{\varphi}=\bar{\psi}+q_{\infty} x_{1} .
$$

We will use the variational approach, which is also successfully applied to the incompressible irrotational Réthy flow problem recently (see [6]), to show the existence of solutions of (2.8). More precisely, let us consider the following variational problem (see [26, 30])

$$
\min _{\bar{\psi} \in \mathcal{V}}\left(\int_{\Omega} \frac{1}{2}|\nabla \bar{\psi}|^{2} d x-q_{\infty} \int_{\Gamma} n_{1} \bar{\psi} d S\right) .
$$

In [30, Ou proved that this minimization problem admits classical solutions and the solution is unique up to an added constant. Indeed, if $\bar{\psi}$ is a solution of the variational problem, then for any $\eta \in C_{c}^{\infty}\left(\mathbb{R}^{n}\right)$, we have

$$
0=\int_{\Omega} \nabla \bar{\psi} \nabla \eta d x+q_{\infty} \int_{\Gamma} n_{1} \eta d S=-\int_{\Omega} \Delta \bar{\psi} \eta d x+\int_{\Gamma}\left(\frac{\partial \bar{\psi}}{\partial \mathbf{n}}+q_{\infty} n_{1}\right) \eta d S .
$$


Thus, $\bar{\psi}$ satisfies

$$
\begin{cases}\Delta \bar{\psi}=0, & x \in \Omega, \\ \frac{\partial \bar{\psi}}{\partial \mathbf{n}}=-q_{\infty} n_{1}, & x \in \Gamma,\end{cases}
$$

in the distributional sense. By the standard elliptic theory, $\bar{\psi}$ is smooth up to the boundary and satisfies (2.9) in the classical sense. Moreover, as $x$ approaches to the infinity, $|\nabla \bar{\psi}|$ tends to zero. Then, the function $\bar{\varphi}=\bar{\psi}+q_{\infty} x_{1}$ satisfies the equation (2.8). In summary, we have the following result.

Theorem 2.2. Problem (2.8) admits a unique classical solution $\bar{\varphi}=\bar{\psi}+q_{\infty} x_{1}$ up to a constant with $\bar{\psi} \in \mathcal{V}$. Here $\mathcal{V}$ is the Hilbert space defined in Definition 2.1. Furthermore,

$$
\int_{\Omega}|\nabla \bar{\psi}|^{2} d x \leq C\left(q_{\infty}, \Omega\right)
$$

and

$$
|\nabla \bar{\psi}|(x) \leq C\left(q_{\infty}, \Omega\right)(1+|x|)^{-\frac{n}{2}}
$$

Finally, we recall that the Bernoulli law for the incompressible irrotational flow is that

$$
\nabla \bar{p}=\nabla\left(\phi-\frac{\left|\bar{u}^{2}\right|}{2}\right)
$$

2.3. Compressible airfoil problem and the low Mach number limit. The irrotational compressible Euler flow with low Mach number can be written as

$$
\left\{\begin{array}{l}
\operatorname{div}\left(\rho^{\varepsilon} u^{\varepsilon}\right)=0, \\
\operatorname{div}\left(\rho^{\varepsilon} u^{\varepsilon} \otimes u^{\varepsilon}\right)+\nabla p^{\varepsilon}=\rho^{\varepsilon} F \\
\operatorname{curl} u^{\varepsilon}=0
\end{array}\right.
$$

with $p^{\varepsilon}=p^{(\varepsilon)}\left(\rho^{\varepsilon}\right)=\frac{\tilde{p}\left(\rho^{\varepsilon}\right)-\tilde{p}(1)}{\varepsilon^{2}}$. And, conservative force $F$ could be written as $F=\nabla \phi$. The Mach number is defined as $M^{\varepsilon}=\frac{\left|u^{\varepsilon}\right|}{\sqrt{\left(p^{(\varepsilon)}\right)^{\prime}\left(\rho^{\varepsilon}\right)}}=\frac{\varepsilon\left|u^{\varepsilon}\right|}{\sqrt{\tilde{p}^{\prime}\left(\rho^{\varepsilon}\right)}}$.

By (2.1)-(2.3), the flow satisfies the slip boundary condition that

$$
u^{\varepsilon} \cdot \vec{n}=0 \text { on } \Gamma,
$$

where $\vec{n}$ denotes the unit inward normal of domain $\mathcal{D}(\Gamma)$, and the asymptotic behavior as $|x| \rightarrow \infty$ that

$$
u^{\varepsilon} \rightarrow\left(q_{\infty}, 0, \cdots, 0\right) \quad \text { and } \quad \rho^{\varepsilon} \rightarrow 1 .
$$

By (2.13), we have the following Bernoulli law that

$$
\nabla\left(\frac{\left|u^{\varepsilon}\right|^{2}}{2}+h^{(\varepsilon)}\left(\rho^{\varepsilon}\right)-\phi\right)=0
$$

where the enthalpy $h^{(\varepsilon)}$ is defined by $h^{(\varepsilon)}(\rho)=\int_{1}^{\rho} \frac{\left(p^{(\varepsilon)}\right)^{\prime}(s)}{s} d s$ up to any constant.

Then by the asymptotic behavior (2.15), we have that

$$
\frac{\left|u^{\varepsilon}\right|^{2}}{2}+h^{(\varepsilon)}\left(\rho^{\varepsilon}\right)=\frac{q_{\infty}^{2}}{2}+h^{(\varepsilon)}(1)+\phi,
$$

Finally, let

$$
\tilde{h}=\varepsilon^{2} h^{(\varepsilon)}
$$


LOW MACH NUMBER LIMIT OF MULTIDIMENSIONAL STEADY FLOWS ON THE AIRFOIL PROBLEM with

$$
\tilde{h}^{\prime}(\rho)=\frac{\tilde{p}^{\prime}(\rho)}{\rho}
$$

By (1.3), we can see that $\tilde{h}(\rho)$ is a strictly increasing function with respect to $\rho$, so does $h^{(\varepsilon)}\left(\rho^{\varepsilon}\right)$. Let

$$
\tilde{H}(\rho):=\frac{\tilde{p}^{\prime}(\rho)}{2}+\tilde{h}(\rho)
$$

Now, we can introduce the critical density $\rho_{c r}^{\varepsilon}$ for each fixed $\varepsilon$ such that

$$
\frac{1}{\varepsilon^{2}} \tilde{H}\left(\rho_{c r}^{\varepsilon}\right)=\frac{q_{\infty}^{2}}{2}+\frac{1}{\varepsilon^{2}} \tilde{h}(1)+\phi .
$$

In this paper, we assume $\phi \in L^{\infty}$. So there exists a constant $\phi^{*}>0$ such that $\phi$ is bounded as

$$
|\phi| \leq \phi^{\star}
$$

Note that

$$
\lim _{\rho \rightarrow+\infty} \tilde{H}(\rho)-\tilde{h}(1)>0>\lim _{\rho \rightarrow 0+} \tilde{H}(\rho)-\tilde{h}(1) .
$$

Then, there exists an $\varepsilon_{0}>0$, such that when $\varepsilon_{0} \geq \varepsilon \geq 0$, it holds

$$
\frac{1}{\varepsilon^{2}}\left(\lim _{\rho \rightarrow+\infty} \tilde{H}(\rho)-\tilde{h}(1)\right)-\frac{q_{\infty}^{2}}{2}>\phi^{*}>\phi>-\phi^{*}>\frac{1}{\varepsilon^{2}}\left(\lim _{\rho \rightarrow 0^{+}} \tilde{H}(\rho)-\tilde{h}(1)\right)-\frac{q_{\infty}^{2}}{2} .
$$

So 2.18 is solvable, and

$$
\rho_{c r}^{\varepsilon}(\phi)=\tilde{H}^{-1}\left(\tilde{h}(1)+\frac{\varepsilon^{2} q_{\infty}^{2}}{2}+\varepsilon^{2} \phi\right)
$$

and the critical speed is

$$
q_{c r}^{\varepsilon}(\phi)=\frac{1}{\varepsilon} \sqrt{\tilde{p}^{\prime} \circ \tilde{H}^{-1}\left(\tilde{h}(1)+\frac{\varepsilon^{2} q_{\infty}^{2}}{2}+\varepsilon^{2} \phi\right)} .
$$

It is easy to see that $\rho_{c r}^{\varepsilon}(\phi) \rightarrow \tilde{H}^{-1}(\tilde{h}(1))$ and $\varepsilon q_{c r}^{\varepsilon}(\phi) \rightarrow \sqrt{\tilde{p}^{\prime} \circ \tilde{H}^{-1}(\tilde{h}(1))}$, so $q_{c r}^{\varepsilon}(\phi)$ will go to the infinity as $\varepsilon$ goes to zero.

It is easy to see that $\left|u^{\varepsilon}\right|<q_{c r}^{\varepsilon}(\phi)$ holds if and only if the flow is subsonic, i.e., $M^{\varepsilon}(\phi)<1$. Similarly, for each $\theta \in(0,1)$, there exists $q_{\theta}^{\varepsilon}(\phi)$ such that $\left|u^{\varepsilon}\right| \leq q_{\theta}^{\varepsilon}(\phi)$ holds if and only if $M^{\varepsilon}(\phi) \leq \theta$. Moreover, $q_{\theta}^{\varepsilon}(\phi)$ is monotonically increasing with respect to $\theta \in(0,1)$. For fixed $\theta \in(0,1), q_{\theta}^{\varepsilon}(\phi) \rightarrow \infty$ as $\varepsilon \rightarrow 0$. Also, for $\varepsilon>0$, both $\varepsilon q_{c r}^{\varepsilon}(\phi)$ and $\varepsilon q_{\theta}^{\varepsilon}(\phi)$ are uniformly bounded respect to $\varepsilon$. Moreover, when $M^{\varepsilon}(\phi)<1$, density $\rho^{\varepsilon}$ can be represented as the function of $\left|u^{\varepsilon}\right|^{2}$ and $\phi$, i.e.,

$$
\begin{aligned}
\rho^{\varepsilon}=\rho^{\varepsilon}\left(\left|u^{\varepsilon}\right|^{2}, \phi\right) & =\left(h^{(\varepsilon)}\right)^{-1}\left(\frac{q_{\infty}^{2}-\left|u^{\varepsilon}\right|^{2}}{2}+h^{(\varepsilon)}(1)+\phi\right) \\
& =\tilde{h}^{-1}\left(\frac{\varepsilon^{2}\left(q_{\infty}^{2}-\left|u^{\varepsilon}\right|^{2}\right)}{2}+\tilde{h}(1)+\varepsilon^{2} \phi\right) .
\end{aligned}
$$

By (2.21), for a given fixed $0<\varepsilon<1$, the flow is subsonic if and only if it holds that

$$
\tilde{H}^{-1}\left(\tilde{h}(1)+\frac{\varepsilon^{2} q_{\infty}^{2}}{2}+\varepsilon^{2} \phi\right)=\rho_{c r}^{\varepsilon}<\rho^{\varepsilon}\left(\left|u^{\varepsilon}\right|^{2}, \phi\right) .
$$


Moreover, by 2.23 ,

$$
\tilde{H}^{-1}\left(\tilde{h}(1)-\phi^{\star}\right)<\rho^{\varepsilon}\left(\left|u^{\varepsilon}\right|^{2}, \phi\right) \leq \tilde{h}^{-1}\left(\frac{\varepsilon^{2} q_{\infty}^{2}}{2}+\tilde{h}(1)+\varepsilon^{2} \phi\right)<\tilde{h}^{-1}\left(\frac{q_{\infty}^{2}}{2}+\tilde{h}(1)+\phi^{\star}\right) .
$$

The low Mach number limit is the limit process when $\varepsilon \rightarrow 0$, that we expect the compressible Euler flow will converges to the corresponding incompressible Euler flow. More precisely, we have the following result which is the main theorem of the paper.

Theorem 2.3. For a given $L^{\infty}$ function $\phi$ which satisfies

$$
\phi \in L^{2}(\Omega) \text { and }\left(1+|x|^{\beta}\right) \nabla \phi \in L^{q}(\Omega) \text { for } q>n, \beta>1-\frac{n}{q},
$$

and for any fixed number $q_{\infty}$, there exists constants $\varepsilon_{c}>0$ and $\alpha \in(0,1)$, such that when $0<\varepsilon<\varepsilon_{c}$ there exists a unique solution $\left(\rho^{\varepsilon}, u^{\varepsilon}, p^{\varepsilon}\right) \in\left(C^{\alpha}(\Omega)\right)^{n+2}$ of Problem 1 corresponding to equations (2.13) with $M^{\varepsilon}<1$. $M^{\varepsilon}$ varies on $(0,1)$ as $\varepsilon$ varies on $\left(0, \varepsilon_{c}\right)$. For any $0<\varepsilon<\varepsilon_{c}$, compressible-incompressible difference velocity $\tilde{u}^{(\varepsilon)} \in\left(C^{\alpha}(\Omega)\right)^{n}$ satisfies:

$$
\left|\tilde{u}^{(\varepsilon)}\right| \leq \frac{C}{(1+|x|)^{\beta^{\prime}}}
$$

where $\beta^{\prime}=\min \left\{\frac{n}{2}, \beta+\frac{n}{q}-1\right\}$. Furthermore, we have that, as $\varepsilon \rightarrow 0$

$$
\rho^{(\varepsilon)}=1+O\left(\varepsilon^{2}\right) \quad u^{(\varepsilon)}=\bar{u}+\varepsilon^{2} \tilde{u}^{(\varepsilon)} \quad \nabla p^{(\varepsilon)}=\nabla \bar{p}+O\left(\varepsilon^{2}\right), \quad M^{\varepsilon}=O(\varepsilon)
$$

where $(1, \bar{u}, \bar{p})$ is the classical solution of Problem 1 corresponding to equations (2.6).

Remark 2.2. If $n=3$, it is easy to check the force potential generated by the solid domain $\Omega^{c}$ (which is the complement of the fluid domain $\Omega$ ):

$$
\phi(x)=\int_{\Omega^{c}} \frac{\rho_{s}(y)}{|x-y|} d y
$$

satisfies the conditions 2.19) and 2.25) for $\phi$, where $x \in \Omega$ and $\rho_{s} \in L^{1}\left(\Omega^{c}\right)$ means the density distribution in $\Omega^{c}$ is of finite mass. As an example, $\phi$ can be the electric field.

Remark 2.3. By (2.25) and the Gagliardo-Nirenberg interpolation inequality, we know that $\phi \in W^{1, q}(\Omega)$ if $n \geq 4$ or $q>6$ if $n=3$. So $\phi$ is an $L^{\infty}$ function by the Morrey's inequality. Hence condition (2.19), and then condition (2.20) follow.

Remark 2.4. In Theorem 2.3, the regularity of $\left(\rho^{(\varepsilon)}, u^{(\varepsilon)}, p^{(\varepsilon)}\right)$ are limited by $\phi$. One can improve the regularity by imposing higher regularity.

Remark 2.5. The effect of conservative force $\nabla \phi$ is very clear here. Since $\bar{u}-q_{\infty} e_{1}$ have the $\frac{n}{2}$ decay rate to infinity, the first order of compressible part $\tilde{u}^{(\varepsilon)}$ may have slower convergence rate due to the decay of $\nabla \phi$.

2.4. Potential formulation of compressible flow. Similar to the incompressible case, we can also introduce the velocity potential $\varphi^{(\varepsilon)}$ for the compressible case that

$$
\nabla \varphi^{(\varepsilon)}=u^{\varepsilon}
$$

with the slip boundary condition that

$$
\frac{\partial \varphi^{\varepsilon}}{\partial \mathbf{n}}=0 \text { on } \Gamma
$$


LOW MACH NUMBER LIMIT OF MULTIDIMENSIONAL STEADY FLOWS ON THE AIRFOIL PROBLEM

where $\mathbf{n}$ denotes the unit inward normal of domain $\mathcal{D}(\Gamma)$, and the asymptotic behavior at the infinity that

$$
\lim _{|x| \rightarrow \infty} \nabla \varphi^{\varepsilon}=\left(q_{\infty}, 0, \cdots, 0\right) .
$$

Therefore, Problem 1 can be reformulated into the following problem.

Problem 2. Let $n \geq 3$. Find function $\varphi^{(\varepsilon)}$ such that

$$
\begin{cases}\operatorname{div}\left(\rho^{\varepsilon}\left(\left|\nabla \varphi^{(\varepsilon)}\right|^{2}, \phi\right) \nabla \varphi^{(\varepsilon)}\right)=0, & x \in \Omega, \\ \frac{\partial \varphi^{(\varepsilon)}}{\partial \mathbf{n}}=0, & x \in \Gamma \\ \lim _{|x| \rightarrow \infty} \nabla \varphi^{(\varepsilon)}=\left(q_{\infty}, 0, \cdots, 0\right) . & \end{cases}
$$

By the straightforward computation, 2.29$)_{1}$ can be rewritten as

$$
\sum_{i j=1}^{n} a_{i j}^{\varepsilon} \partial_{i j} \varphi^{(\varepsilon)}+\sum_{i=1}^{n} b_{i}^{\varepsilon} \partial_{i} \varphi^{(\varepsilon)}=0,
$$

where

$$
a_{i j}^{\varepsilon}=\rho^{\varepsilon}\left(\delta_{i j}-\frac{\varepsilon^{2} \partial_{i} \varphi^{(\varepsilon)} \partial_{j} \varphi^{(\varepsilon)}}{\tilde{p}^{\prime}\left(\rho^{\varepsilon}\right)}\right) \text {, }
$$

and

$$
b_{i}^{\varepsilon}=\frac{\varepsilon^{2} \rho^{\varepsilon} \partial_{i} \phi}{\tilde{p}^{\prime}\left(\rho^{\varepsilon}\right)} .
$$

For $0<\varepsilon<1$ and $M^{\varepsilon}<\theta<1$, we have that

$$
0<\lambda_{1}|\xi|^{2} \leq \sum_{i j=1}^{n} a_{i j}^{\varepsilon} \xi_{i} \xi_{j} \leq \lambda_{2}|\xi|^{2},
$$

where constants $\lambda_{1}$ and $\lambda_{2}$ do not depend on $\varepsilon$.

\section{Variational Approach of the Compressible airfoil problem}

To show Theorem 2.3, we should first show the existence of smooth solution of the compressible airfoil problem. To show it, in this section, we will first introduce a variational formulation, and then show the existence of the minimizer which is a solution of the compressible airfoil problem.

3.1. A variational formulation for the compressible flow. Notice that equation 2.30) is nonlinear and is uniformly elliptic if and only if $M^{\varepsilon}<\theta<1$. Therefore we need to introduce the cut-off to truncate the coefficients of equation 2.30 . For $0<\varepsilon_{0}<1$, $0<\theta<1$, we introduce $\stackrel{\stackrel{\varepsilon}{q}}{\theta}^{\varepsilon_{0}}(\phi)=\inf _{0<\varepsilon<\varepsilon_{0}} q_{\theta}^{\varepsilon}(\phi)$, and cut-off function on the phase plan

$$
\hat{q}\left(q^{2}, \phi\right)= \begin{cases}q^{2}-2 \phi & \text { if }|q| \leq \stackrel{\circ}{q}_{\theta}^{\varepsilon_{0}}(\phi), \\ \text { monotone smooth function } & \text { if } q_{\theta}^{\varepsilon_{0}}(\phi) \leq|q| \leq \stackrel{\circ}{q}_{\frac{\theta+1}{2}}^{\varepsilon_{0}}(\phi), \\ \sup _{x \in \Omega}\left(\left(\stackrel{\circ}{q}_{\frac{\theta+1}{2}}^{\varepsilon_{0}}\right)^{2}(\phi)-2 \phi\right)(x) & \text { if }|q| \geq \stackrel{\circ}{q}_{\frac{\theta+1}{2}}^{\varepsilon_{0}}(\phi),\end{cases}
$$

Let $\hat{\rho}^{(\varepsilon)}$ satisfy

$$
\frac{\hat{q}\left(q^{2}, \phi\right)}{2}+h^{(\varepsilon)}\left(\hat{\rho}^{(\varepsilon)}\right)=\frac{q_{\infty}^{2}}{2}+h^{(\varepsilon)}(1)
$$


which is equivalent to that

$$
\hat{\rho}^{\varepsilon}=\hat{\rho}^{\varepsilon}\left(q^{2}, \phi\right)=\tilde{h}^{-1}\left(\frac{\varepsilon^{2}\left(q_{\infty}^{2}-\hat{q}\left(q^{2}, \phi\right)\right)}{2}+\tilde{h}(1)\right) .
$$

We remark that in this paper, we will construct solutions that satisfy $|\nabla \bar{\varphi}| \leq \stackrel{\circ}{q}_{\theta}^{\varepsilon_{0}}$, which implies $\hat{q}\left(|\nabla \bar{\varphi}|^{2}\right)=|\nabla \bar{\varphi}|^{2}$. Moreover, define $\stackrel{q}{c r}_{c}^{\varepsilon_{0}}=\inf _{0<\varepsilon<\varepsilon_{0}} q_{c r}^{\varepsilon}$. And, we denote $\hat{\rho}_{\Lambda}^{\varepsilon}(\Lambda, \phi):=\frac{\partial}{\partial \Lambda} \hat{\rho}^{\varepsilon}(\Lambda, \phi)$, and $\hat{\rho}_{\phi}^{\varepsilon}(\Lambda, \phi):=\frac{\partial}{\partial \phi} \hat{\rho}^{\varepsilon}(\Lambda, \phi)$.

Then, Problem 2 is reformulated into Problem 3 as follows.

Problem 3: Let $n \geq 3$. Find function $\varphi^{(\varepsilon)}$ which satisfies

$$
\begin{cases}\operatorname{div}\left(\hat{\rho}^{\varepsilon}\left(\left|\nabla \varphi^{(\varepsilon)}\right|^{2}, \phi\right) \nabla \varphi^{(\varepsilon)}\right)=0, & x \in \Omega, \\ \frac{\partial \varphi^{(\varepsilon)}}{\partial \mathbf{n}}=0, & x \in \Gamma, \\ \lim _{|x| \rightarrow \infty} \nabla \varphi^{(\varepsilon)}=\left(q_{\infty}, 0, \cdots, 0\right) . & \end{cases}
$$

Straightforward calculation yields that $(3.3)_{1}$ can be rewritten as

$$
\sum_{i, j=1}^{n} \hat{a}_{i j}\left(\nabla \varphi^{(\varepsilon)}, \phi\right) \partial_{i j} \varphi^{(\varepsilon)}+\sum_{i=1}^{n} \hat{b}_{i}\left(\nabla \varphi^{(\varepsilon)}, \phi\right) \partial_{i} \varphi^{(\varepsilon)}=0
$$

where

$$
\begin{aligned}
\hat{a}_{i j}\left(\nabla \varphi^{(\varepsilon)}, \phi\right) & =\hat{\rho}^{\varepsilon}\left(\left|\nabla \varphi^{(\varepsilon)}\right|^{2}, \phi\right)\left(\delta_{i j}-\frac{\hat{q}_{\Lambda}\left(\left|\nabla \varphi^{(\varepsilon)}\right|^{2}, \phi\right) \partial_{i} \varphi^{(\varepsilon)} \partial_{j} \varphi^{(\varepsilon)}}{\left(c^{\varepsilon}\right)^{2}}\right) \\
& =\hat{\rho}^{\varepsilon}\left(\left|\nabla \varphi^{(\varepsilon)}\right|^{2}\right)\left(\delta_{i j}-\frac{\varepsilon^{2} \hat{q}_{\Lambda}\left(\left|\nabla \varphi^{(\varepsilon)}\right|^{2}, \phi\right) \partial_{i} \varphi^{(\varepsilon)} \partial_{j} \varphi^{(\varepsilon)}}{\tilde{p}^{\prime}\left(\hat{\rho}^{\varepsilon}\right)}\right),
\end{aligned}
$$

and

$$
\hat{b}_{i}\left(\nabla \varphi^{(\varepsilon)}, \phi\right)=\frac{\varepsilon^{2} \hat{\rho}^{\varepsilon} \hat{q}_{\phi}\left(\left|\nabla \varphi^{(\varepsilon)}\right|^{2}, \phi\right) \partial_{i} \phi}{\tilde{p}^{\prime}\left(\hat{\rho}^{\varepsilon}\right)}
$$

Moreover,

$$
\hat{\lambda}_{1}|\xi|^{2} \leq \sum_{i, j=1}^{n} \hat{a}_{i j}\left(\nabla \varphi^{(\varepsilon)}, \phi\right) \xi_{i} \xi_{j} \leq \hat{\lambda}_{2}|\xi|^{2}, \quad\left|\hat{b}_{i} \partial_{i} \varphi^{(\varepsilon)}\right| \leq C\left|\partial_{i} \phi\right|,
$$

where constants $C, \hat{\lambda}_{1}$, and $\hat{\lambda}_{2}$ depend only on the subsonic truncation parameters $\theta$ and $\varepsilon_{0}$, and do not depend on solution $\varphi^{(\varepsilon)}$.

Finally, we remark that a solution of Problem 3, where the density $\hat{\rho}^{(\varepsilon)}$ is derived from the new density-speed relation (3.1), is also a solution of the original potential flow equation in the Problem 2 when $\left|\nabla \varphi^{(\varepsilon)}\right|<\stackrel{\circ}{q}_{\theta}^{\varepsilon_{0}}$.

To solve Problem 3, we follow the idea used in [8] to introduce a variational formulation. Denote

$$
G^{(\varepsilon)}(\Lambda, \phi)=\frac{1}{2} \int_{0}^{\Lambda} \hat{\rho}^{(\varepsilon)}(\lambda, \phi) d \lambda
$$

Formally, 3.3 is the Euler-Lagrangian equation of the variational problem with respect to the integral that

$$
\int_{\Omega} G^{(\varepsilon)}\left(\left|\nabla \varphi^{(\varepsilon)}\right|^{2}, \phi\right) d x
$$


LOW MACH NUMBER LIMIT OF MULTIDIMENSIONAL STEADY FLOWS ON THE AIRFOIL PROBLEM

However, the integral above is infinite due to the unbounded domain $\Omega$. To overcome it, we introduce

$$
I^{(\varepsilon)}(\varphi, \bar{\varphi})=\varepsilon^{-4} \int_{\Omega}\left[G^{(\varepsilon)}\left(|\nabla \varphi|^{2}, \phi\right)-G^{(\varepsilon)}\left(|\nabla \bar{\varphi}|^{2}, \phi\right)-\nabla \bar{\varphi} \cdot(\nabla \varphi-\nabla \bar{\varphi})\right] d x .
$$

Then Problem 3 becomes:

Problem 4 : Find a minimizer $\tilde{\varphi}^{(\varepsilon)} \in \mathcal{V}$ such that

$$
I^{(\varepsilon)}\left(\bar{\varphi}+\varepsilon^{2} \tilde{\varphi}^{(\varepsilon)}, \bar{\varphi}\right)=\min _{\tilde{\varphi} \in \mathcal{V}} I^{(\varepsilon)}\left(\bar{\varphi}+\varepsilon^{2} \tilde{\varphi}, \bar{\varphi}\right) .
$$

Here, $\tilde{\varphi}=\frac{\varphi-\bar{\varphi}}{\varepsilon^{2}}$ and $\tilde{\varphi}^{(\varepsilon)}=\frac{\varphi^{(\varepsilon)}-\bar{\varphi}}{\varepsilon^{2}}$.

Remark 3.1. The minimizer of Problem 4 satisfies (3.3).

Proof. Let $\eta \in C_{c}^{\infty}\left(\mathbb{R}^{n}\right)$. The first variation of $I^{(\varepsilon)}\left(\varphi^{(\varepsilon)}, \bar{\varphi}\right)$ with $\eta$ is

$$
0=\int_{\Omega}\left[2 G_{\Lambda}^{(\varepsilon)}\left(\left|\nabla \varphi^{(\varepsilon)}\right|^{2}, \phi\right) \nabla \varphi^{(\varepsilon)} \cdot \nabla \eta-\nabla \bar{\varphi} \cdot \nabla \eta\right] d x
$$

The second term in the integrand above vanishes due to the definition of the incompressible flow $\bar{\varphi}$. Then, the first variation of $I^{(\varepsilon)}\left(\varphi^{(\varepsilon)}, \bar{\varphi}\right)$ associated with $\eta$ is

$$
\begin{aligned}
0 & =\int_{\Omega} 2 G_{\Lambda}^{(\varepsilon)}\left(\left|\nabla \varphi^{(\varepsilon)}\right|^{2}, \phi\right) \nabla \varphi^{(\varepsilon)} \cdot \nabla \eta d x \\
& =\int_{\Omega} \tilde{\rho}^{(\varepsilon)}\left(\left|\nabla \varphi^{(\varepsilon)}\right|^{2}, \phi\right) \nabla \varphi^{(\varepsilon)} \cdot \nabla \eta d x
\end{aligned}
$$

Hence this remark follows from the identity above.

3.2. Unique existence of the minimizer of Problem 4. First, for the existence of a minimizer of Problem 4, we have the following theorem.

Theorem 3.1. Problem 4 admits a unique minimizer $\tilde{\varphi}^{(\varepsilon)} \in \mathcal{V}$, which satisfies that

$$
\int_{\Omega}\left|\nabla \tilde{\varphi}^{(\varepsilon)}\right|^{2} d x \leq C
$$

where constant $C$ depends only on $\bar{\varphi}$ and $\Omega$ and does not depend on $\varepsilon$.

Proof. The proof is divided into four steps.

Step 1. $I^{(\varepsilon)}\left(\bar{\varphi}+\varepsilon^{2} \tilde{\varphi}, \bar{\varphi}\right)=I^{(\varepsilon)}(\varphi, \bar{\varphi})$ is coercive with respect to $\tilde{\varphi}$ in $\mathcal{V}$, i.e., we will show that

$$
I^{(\varepsilon)}(\varphi, \bar{\varphi}) \geq \frac{C_{1}}{2} \int_{\Omega}|\nabla \tilde{\varphi}|^{2} d x-C_{3} \int_{\Omega}\left|\nabla \bar{\varphi}-e_{1} q_{\infty}\right|^{2} d x-C \int_{\Omega}|\phi|^{2} d x .
$$

Let

$$
\begin{aligned}
& I_{1}^{(\varepsilon)}(\varphi, \bar{\varphi}) \\
:= & \varepsilon^{-4} \int_{\Omega}\left[G^{(\varepsilon)}\left(|\nabla \varphi|^{2}, \phi\right)-G^{(\varepsilon)}\left(|\nabla \bar{\varphi}|^{2}, \phi\right)-2 G_{\Lambda}^{(\varepsilon)}\left(|\nabla \bar{\varphi}|^{2}, \phi\right) \nabla \bar{\varphi} \cdot(\nabla \varphi-\nabla \bar{\varphi})\right] d x,
\end{aligned}
$$

and

$$
I_{2}^{(\varepsilon)}(\varphi, \bar{\varphi}):=\varepsilon^{-4} \int_{\Omega}\left[2\left(G_{\Lambda}^{(\varepsilon)}\left(|\nabla \bar{\varphi}|^{2}, \phi\right)-1\right) \nabla \bar{\varphi} \cdot(\nabla \varphi-\nabla \bar{\varphi})\right] d x .
$$

Obviously, $I^{(\varepsilon)}(\varphi, \bar{\varphi})=I_{1}^{(\varepsilon)}(\varphi, \bar{\varphi})+I_{2}^{(\varepsilon)}(\varphi, \bar{\varphi})$. 
First, we will show that $I_{1}^{(\varepsilon)}(\varphi, \bar{\varphi})$ is coercive with respect to $\tilde{\varphi}$ in $\mathcal{V}$.

We denote $p=\left(p_{1}, \cdots, p_{n}\right), F^{(\varepsilon)}(p)=G^{(\varepsilon)}\left(|p|^{2}, \phi\right)$. Then by direct computation, we can get that

$$
\begin{aligned}
& G^{(\varepsilon)}\left(|\nabla \varphi|^{2}, \phi\right)-G^{(\varepsilon)}\left(|\nabla \bar{\varphi}|^{2}, \phi\right)-2 G_{\Lambda}^{(\varepsilon)}\left(|\nabla \bar{\varphi}|^{2}, \phi\right) \nabla \bar{\varphi} \cdot(\nabla \varphi-\nabla \bar{\varphi}) \\
= & F^{(\varepsilon)}(\nabla \varphi)-F^{(\varepsilon)}(\nabla \bar{\varphi})-\nabla F^{(\varepsilon)}(\nabla \bar{\varphi}) \cdot(\nabla \varphi-\nabla \bar{\varphi}) \\
= & \sum_{i, j=1}^{n} \int_{0}^{1}(1-t) \partial_{p_{i} p_{j}} F^{(\varepsilon)}(t \nabla \varphi+(1-t) \nabla \bar{\varphi}) d t \partial_{i}(\varphi-\bar{\varphi}) \partial_{j}(\varphi-\bar{\varphi}) .
\end{aligned}
$$

It is easy to check $\partial_{p p}^{2} F^{(\varepsilon)}$ is uniformly positive. In fact, we have

$$
\left(\partial_{p p}^{2} F^{(\varepsilon)}(p)\right)_{i, j}=\hat{a}_{i j}\left(\nabla \varphi^{(\varepsilon)}, \phi\right) .
$$

From property (3.6), we get the uniformly positivity of $\partial_{p p}^{2} F^{(\varepsilon)}$. As a consequence, we have

$$
\frac{C_{1}}{2} \int_{\Omega}|\nabla \tilde{\varphi}|^{2} d x \leq I_{1}^{(\varepsilon)}(\varphi, \bar{\varphi}) \leq \frac{\tilde{C}_{1}}{2} \int_{\Omega}|\nabla \tilde{\varphi}|^{2} d x .
$$

Now, let us consider $I_{2}^{(\varepsilon)}(\varphi, \bar{\varphi})$. Note that

$$
\begin{aligned}
\left|\varepsilon^{-2}\left(2 G_{\Lambda}^{(\varepsilon)}\left(|\nabla \bar{\varphi}|^{2}, \phi\right)-1\right)\right| & =\left|\varepsilon^{-2}\left(\hat{\rho}^{(\varepsilon)}\left(|\nabla \bar{\varphi}|^{2}, \phi\right)-1\right)\right| \\
& =\left|\varepsilon^{-2}\left(\tilde{h}^{-1}\left(\frac{\varepsilon^{2}\left(q_{\infty}^{2}-\hat{q}\left(|\nabla \bar{\varphi}|^{2}, \phi\right)\right)}{2}+\tilde{h}(1)\right)-1\right)\right| \\
& =\left|\varepsilon^{-2}\left(\tilde{h}^{-1}\left(\frac{\varepsilon^{2}\left(q_{\infty}^{2}-|\nabla \bar{\varphi}|^{2}+2 \phi\right)}{2}+\tilde{h}(1)\right)-\tilde{h}^{-1}(\tilde{h}(1))\right)\right| \\
& =\left|\frac{q_{\infty}^{2}-|\nabla \bar{\varphi}|^{2}+2 \phi}{2} \int_{0}^{1}\left(\tilde{h}^{-1}\right)^{\prime}\left(t \frac{\varepsilon^{2}\left(q_{\infty}^{2}-|\nabla \bar{\varphi}|^{2}+2 \phi\right)}{2}+\tilde{h}(1)\right) d t\right| \\
\leq & \left(\left|\nabla \bar{\varphi}-e_{1} q_{\infty}\right|\left|\frac{q_{\infty}+|\nabla \bar{\varphi}|}{2}\right|+|\phi|\right) \\
& \left|\int_{0}^{1}\left(\tilde{h}^{-1}\right)^{\prime}\left(t \frac{\varepsilon^{2}\left(q_{\infty}^{2}-|\nabla \bar{\varphi}|^{2}+2 \phi\right)}{2}+\tilde{h}(1)\right) d t\right| .
\end{aligned}
$$

Then

$$
\begin{aligned}
\left|I_{2}^{(\varepsilon)}(\varphi, \bar{\varphi})\right| & =\left|\varepsilon^{-4} \int_{\Omega}\left[\left(2 G_{\Lambda}^{(\varepsilon)}\left(|\nabla \bar{\varphi}|^{2}, \phi\right)-1\right) \nabla \bar{\varphi} \cdot(\nabla \varphi-\nabla \bar{\varphi})\right] d x\right| \\
& \leq C \varepsilon^{-2} \int_{\Omega}\left(\left|\nabla \bar{\varphi}-e_{1} q_{\infty}\right|+|\phi|\right)|\nabla(\varphi-\bar{\varphi})| d x \\
& \leq C \int_{\Omega}\left|\nabla \bar{\varphi}-e_{1} q_{\infty}\right|^{2} d x+C \int_{\Omega}|\phi|^{2} d x+\frac{C_{1}}{4} \varepsilon^{-4} \int_{\Omega}|\nabla(\varphi-\bar{\varphi})|^{2} d x .
\end{aligned}
$$

By (3.14), (3.15), and the definition of $\tilde{\varphi}$ together, we get (3.11), which also implies that $I^{(\bar{\varepsilon})}(\varphi, \bar{\varphi})$ is bounded from below, i.e., there exists a constant $C>0$ depending only on the data such that

$$
I^{(\varepsilon)}(\varphi, \bar{\varphi}) \geq-C
$$

for all $\tilde{\varphi} \in \mathcal{V}$. 
LOW MACH NUMBER LIMIT OF MULTIDIMENSIONAL STEADY FLOWS ON THE AIRFOIL PROBLEN及

Step 2. Similarly, by (3.14) and (3.15) together, we can also show the upper bound of $I^{(\varepsilon)}(\varphi, \bar{\varphi})$ as:

$$
I^{(\varepsilon)}(\varphi, \bar{\varphi}) \leq \frac{C_{1}+2 \tilde{C}_{1}}{2} \int_{\Omega}|\nabla \tilde{\varphi}|^{2} d x+C_{3} \int_{\Omega}\left|\nabla \bar{\varphi}-e_{1} q_{\infty}\right|^{2} d x+C_{4} \int_{\Omega}|\phi|^{2} d x
$$

It means that $I^{(\varepsilon)}(\varphi, \bar{\varphi})=I^{(\varepsilon)}\left(\bar{\varphi}+\varepsilon^{2} \tilde{\varphi}, \bar{\varphi}\right)$ is finite for any $\tilde{\varphi} \in \mathcal{V}$.

Step 3. We will prove $I^{(\varepsilon)}(\varphi, \bar{\varphi})$ is uniformly convex in the space $\mathcal{V}$.

Since $I_{2}^{(\varepsilon)}(\varphi, \bar{\varphi})$ is linear respect to $\tilde{\varphi}$. for any $\tilde{\varphi}_{1}, \tilde{\varphi}_{2} \in \mathcal{V}$, we have that

$$
\begin{aligned}
& I^{(\varepsilon)}\left(\varphi_{1}, \bar{\varphi}\right)+I^{(\varepsilon)}\left(\varphi_{2}, \bar{\varphi}\right)-2 I^{(\varepsilon)}\left(\frac{\varphi_{1}+\varphi_{2}}{2}, \bar{\varphi}\right) \\
= & I_{1}^{(\varepsilon)}\left(\varphi_{1}, \bar{\varphi}\right)+I_{1}^{(\varepsilon)}\left(\varphi_{2}, \bar{\varphi}\right)-2 I_{1}^{(\varepsilon)}\left(\frac{\varphi_{1}+\varphi_{2}}{2}, \bar{\varphi}\right) \\
= & \int_{\Omega} F\left(\nabla \varphi_{1}\right)+F\left(\nabla \varphi_{2}\right)-2 F\left(\frac{\nabla \varphi_{1}+\nabla \varphi_{2}}{2}\right) d x \\
\geq & \frac{C_{1}}{2} \varepsilon^{-4}\left\|\varphi_{1}-\varphi_{2}\right\|_{\mathcal{V}}^{2}=\frac{C_{1}}{2}\left\|\tilde{\varphi}_{1}-\tilde{\varphi}_{2}\right\|_{\mathcal{V}}^{2},
\end{aligned}
$$

which proves the uniform convexity of $I^{(\varepsilon)}$.

Step 4. We are ready to show the unique existence of minimizer $\tilde{\varphi}^{(\varepsilon)} \in \mathcal{V}$ of Problem 4, which satisfies 3.10 .

Firstly, we show the continuity of $I^{(\varepsilon)}\left(\bar{\varphi}+\varepsilon^{2} \tilde{\varphi}, \bar{\varphi}\right)$ with respect to $\tilde{\varphi}$ in $\mathcal{V}$. Taking $\tilde{\varphi}_{1}$ and $\tilde{\varphi}_{2}$ in $\mathcal{V}$, corresponding to $\varphi_{1}$ and $\varphi_{2}$ respectively, we have

$$
\begin{aligned}
I^{(\varepsilon)}\left(\varphi_{1}, \bar{\varphi}\right)-I^{(\varepsilon)}\left(\varphi_{2}, \bar{\varphi}\right)= & \varepsilon^{-4} \int_{\Omega}\left[\frac{1}{2} \int_{\left|\nabla \varphi_{2}\right|^{2}}^{\left|\nabla \varphi_{1}\right|^{2}} \hat{\rho}^{(\varepsilon)}(\Lambda, \phi) d \Lambda-\nabla \bar{\varphi} \cdot \nabla\left(\varphi_{1}-\varphi_{2}\right)\right] d x \\
= & \varepsilon^{-4} \int_{\Omega}\left[\frac{1}{2} \int_{\left|\nabla \varphi_{2}\right|^{2}}^{\left|\nabla \varphi_{1}\right|^{2}} \hat{\rho}^{(\varepsilon)}(\Lambda, \phi) d \Lambda-\hat{\rho}^{(\varepsilon)}\left(|\nabla \bar{\varphi}|^{2}\right) \nabla \bar{\varphi} \cdot \nabla\left(\varphi_{1}-\varphi_{2}\right)\right] d x \\
& +\varepsilon^{-2} \int_{\Omega}\left[\varepsilon^{-2}\left(\hat{\rho}^{(\varepsilon)}\left(|\nabla \bar{\varphi}|^{2}, \phi\right)-1\right) \nabla \bar{\varphi} \cdot \nabla\left(\varphi_{1}-\varphi_{2}\right)\right] d x
\end{aligned}
$$

By a similar argument as done in Step 1 to obtain (3.14) and (3.15), and by the Hölder inequality, we have:

$$
\begin{aligned}
& \left|I^{(\varepsilon)}\left(\varphi_{1}, \bar{\varphi}\right)-I^{(\varepsilon)}\left(\varphi_{2}, \bar{\varphi}\right)\right| \\
\leq & \frac{\tilde{C}_{1}}{2} \int_{\Omega}\left|\nabla\left(\tilde{\varphi}_{1}-\tilde{\varphi}_{2}\right)\right|^{2} d x+C \varepsilon^{-2} \int_{\Omega}\left(\left|\nabla \varphi-e_{1} q_{\infty}\right|+|\phi|\right)\left|\nabla\left(\varphi_{1}-\varphi_{2}\right)\right| d x \\
\leq & C\left\|\tilde{\varphi}_{1}-\tilde{\varphi}_{2}\right\|_{\mathcal{V}}^{2}+C\left\|\tilde{\varphi}_{1}-\tilde{\varphi}_{2} \mid\right\|_{\mathcal{V}} .
\end{aligned}
$$

Now we can show the existence of the minimizer $\tilde{\varphi}^{(\varepsilon)}$ by the compactness argument via applying the continuity of the functional $I^{(\varepsilon)}\left(\bar{\varphi}+\varepsilon^{2} \tilde{\varphi}, \bar{\varphi}\right)$ with respect to $\tilde{\varphi}$ in $\mathcal{V}$.

For the uniqueness, by (3.18), we know that if $\varphi$ is the minimizer, then for any $\varphi_{*} \in \mathcal{V}$, we have that

$$
I^{(\varepsilon)}\left(\varphi_{*}, \bar{\varphi}\right)-I^{(\varepsilon)}(\varphi, \bar{\varphi}) \geq \frac{C_{1}}{2} \varepsilon^{-4}\left\|\varphi_{*}-\varphi\right\|_{\mathcal{V}}^{2} .
$$


Therefore,

$$
I^{(\varepsilon)}\left(\varphi_{*}, \bar{\varphi}\right)>I^{(\varepsilon)}(\varphi, \bar{\varphi}),
$$

for any $\varphi_{*} \in \mathcal{V}$ and $\varphi_{*} \neq \varphi$. It means that the minimizer is unique in $\mathcal{V}$.

Finally, (3.10) easily follows from (3.11) via replacing $\varphi$ by $\bar{\varphi}$, since we know that $I^{(\varepsilon)}(\varphi, \bar{\varphi}) \leq I^{(\varepsilon)}(\bar{\varphi}, \bar{\varphi})$.

\section{Proof of Theorem 2.3}

By Remark 3.1, we know that the minimizer of Problem 4 is a weak solution of Problem 3. In this section, we will first show the minimizer of Problem 4 is a solution of Problem 2 by remove the elliptic cut-off introduced in Problem 3, and then show the low Mach number limit to conclude the proof of Theorem 2.3 .

4.1. $C^{1, \alpha}$-Regularity of the minimizer. Before removing the elliptic cut-off, let us consider the regularity of the derivatives of the solutions.

Firstly, we need the following proposition.

Proposition 4.1. Let $a_{i j}^{l}$ for $i, j=1, \ldots, n$ be $L^{\infty}$ functions on $B_{1}$, and $\lambda$ be a positive constant. Assume that

$$
\forall \xi \in \mathbb{R}^{n}, \lambda|\xi|^{2} \leq \sum_{i, j=1}^{n} a_{i j}^{l} \xi_{i} \xi_{j} \leq \lambda^{-1}|\xi|^{2}, \text { and } f_{i}^{l} \in L^{q}, q>n .
$$

Let $w(y)$ be a function in $H^{1}$. Suppose

$$
\sum_{i, j=1}^{n} \partial_{i}\left[a_{i j}^{l}(y) \partial_{j} w(y)\right]+\sum_{i=1}^{n} \partial_{i} f_{i}^{l}=0
$$

holds in the distribution sense. Then $w(y)$ is Hölder continuous in $B_{1 / 2}$ and there exist two constants $0<\alpha \leq 1, k$, depending on $\lambda$ such that

$$
\begin{gathered}
\sup _{y \in B_{1 / 2}}|w(y)| \leq k\left(\|w\|_{L^{2}\left(B_{1}\right)}+\left\|f_{i}^{l}\right\|_{L^{q}\left(B_{1}\right)}\right), \\
\sup _{y_{1}, y_{2} \in B_{1 / 2}} \frac{\left|w\left(y_{1}\right)-w\left(y_{2}\right)\right|}{\left|y_{1}-y_{2}\right|^{\alpha}} \leq k\left(\|w\|_{L^{2}\left(B_{1}\right)}+\left\|f_{i}^{l}\right\|_{L^{q}\left(B_{1}\right)}\right) .
\end{gathered}
$$

The proof of this proposition can be found in [16] (see Theorem 8.24).

Based on Proposition 4.1, we can show the $C^{\alpha}$-regularity of $\nabla \tilde{\varphi}$.

Lemma 4.1. The minimizer $\tilde{\varphi}^{(\varepsilon)}$ of Problem 4 satisfies that

$$
\left|\nabla \tilde{\varphi}^{(\varepsilon)}\right| \leq \frac{C}{(1+|x|)^{\beta^{\prime}}},
$$

where $\beta^{\prime}=\min \left\{\frac{n}{2}, \beta+\frac{n}{q}-1\right\}$, and that

$$
\left\|\nabla \tilde{\varphi}^{(\varepsilon)}\right\|_{C^{\alpha}(\Omega)} \leq C,
$$

where constant $C$ is independent of $\varepsilon$. 
LOW MACH NUMBER LIMIT OF MULTIDIMENSIONAL STEADY FLOWS ON THE AIRFOIL PROBLEM

Proof. We divide the proof into three steps.

Step 1. Let $\Phi=\partial_{k} \tilde{\varphi}^{(\varepsilon)}, \bar{\varphi}^{\prime}=\partial_{k} \bar{\varphi}$ for $k=1, \ldots, n$. Then by the straightforward calculation, $\Phi$ satisfies

$$
\sum_{i, j=1}^{n} \partial_{i}\left(\hat{a}_{i j} \partial_{j} \Phi\right)+\sum_{i=1} \partial_{i}\left(\hat{b}_{k} \partial_{i} \varphi^{(\varepsilon)}\right)+\varepsilon^{-2} \sum_{i, j=1}^{n} \partial_{i}\left(\hat{a}_{i j} \partial_{j} \bar{\varphi}^{\prime}\right)=0 .
$$

Since $\Delta \bar{\varphi}=0, \Delta \bar{\varphi}^{\prime}=0$. We can change the equation above to:

$$
\sum_{i, j=1}^{n} \partial_{i}\left(\hat{a}_{i j} \partial_{j} \Phi\right)=-\varepsilon^{-2} \sum_{i, j=1}^{n} \partial_{i}\left(\left(\hat{a}_{i j}-\delta_{i j}\right) \partial_{j} \bar{\varphi}^{\prime}\right)-\sum_{i=1} \partial_{i}\left(\hat{b}_{k} \partial_{i} \varphi^{(\varepsilon)}\right) .
$$

Here, we introduce

$$
\begin{aligned}
f_{i j} & =\varepsilon^{-2}\left(\hat{a}_{i j}-\delta_{i j}\right) \\
& =\frac{\hat{\rho}^{(\varepsilon)}-1}{\varepsilon^{2}} \delta_{i j}-\varepsilon^{-2} \frac{\hat{q}_{\Lambda}\left(\left|\nabla \varphi^{(\varepsilon)}\right|^{2}, \phi\right) \partial_{i} \varphi^{(\varepsilon)} \partial_{j} \varphi^{(\varepsilon)}}{\left(c^{\varepsilon}\right)^{2}},
\end{aligned}
$$

then,

$$
\varepsilon^{-2} \sum_{i, j=1}^{n} \partial_{i}\left(\left(\hat{a}_{i j}-\delta_{i j}\right) \partial_{j} \bar{\varphi}^{\prime}\right)=\sum_{i=1}^{n} \partial_{i}\left(\sum_{j=1}^{n} f_{i j} \partial_{j} \bar{\varphi}^{\prime}\right)
$$

Now we are going to show the uniform $L^{\infty}$ estimate of $f_{i j}$. For the first term,

$$
\begin{aligned}
\frac{\hat{\rho}^{(\varepsilon)}-1}{\varepsilon^{2}} & =\frac{\hat{\rho}^{(\varepsilon)}\left(\left|\nabla \varphi^{(\varepsilon)}\right|^{2}, \phi\right)-1}{\varepsilon^{2}} \\
& =\frac{\tilde{h}^{-1}\left(\frac{\varepsilon^{2}\left(q_{\infty}^{2}-\left(\hat{q}\left(\left|\nabla \varphi^{(\varepsilon)}\right|^{2}, \phi\right)\right)\right.}{2}+\tilde{h}(1)\right)-1}{\varepsilon^{2}} \\
& =\frac{\tilde{h}^{-1}\left(\frac{\varepsilon^{2}\left(q_{\infty}^{2}-\left(\hat{q}\left(\left|\nabla \varphi^{(\varepsilon)}\right|^{2}, \phi\right)\right)\right)}{2}+\tilde{h}(1)\right)-\tilde{h}^{-1}(h(1))}{\varepsilon^{2}} \\
& =\frac{q_{\infty}^{2}-\hat{q}\left(\left|\nabla \varphi^{(\varepsilon)}\right|^{2}, \phi\right)}{2} \int_{0}^{1}\left(\tilde{h}^{-1}\right)^{\prime}\left(t \varepsilon^{2} \frac{q_{\infty}^{2}-\hat{q}\left(\left|\nabla \varphi^{(\varepsilon)}\right|^{2}, \phi\right)}{2}+\tilde{h}(1)\right) d t
\end{aligned}
$$

For the second term,

$$
\varepsilon^{-2} \frac{\hat{q}_{\Lambda}\left(\left|\nabla \varphi^{(\varepsilon)}\right|^{2}, \phi\right) \partial_{i} \varphi^{(\varepsilon)} \partial_{j} \varphi^{(\varepsilon)}}{\left(c^{\varepsilon}\right)^{2}}=\frac{\hat{q}_{\Lambda}\left(\left|\nabla \varphi^{(\varepsilon)}\right|^{2}, \phi\right) \partial_{i} \varphi^{(\varepsilon)} \partial_{j} \varphi^{(\varepsilon)}}{\tilde{p}^{\prime}\left(\rho^{\varepsilon}\right)} .
$$

Therefore, due to the cut-off, we have the uniform $L^{\infty}$ estimate of $f_{i j}$.

Also, for $i, k=1, \cdots, n$,

$$
\left|\hat{b}_{k} \partial_{i} \varphi^{(\varepsilon)}\right| \leq C\left|\partial_{k} \phi\right|
$$

By the assumption that $\nabla \phi \in L^{q}$, we know $\hat{b}_{i} \partial_{i} \varphi^{(\varepsilon)}$ are bounded in $L^{q}$ for $q>n$.

Step 2. Now we can show the interior estimates based on the equations derived in Step 1. For any bounded interior subregion of $\Omega$, since $\bar{\varphi}^{\prime}$ satisfies $\Delta \bar{\varphi}^{\prime}=0$, and $\|\nabla \bar{\varphi}\|_{L^{2}}$ is uniformly bounded, from the standard elliptic estimate, we have that $\partial_{j} \bar{\varphi}^{\prime}$ is locally $C^{\infty}$ and uniformly bounded, i.e.,

$$
\left\|\partial_{i} \bar{\varphi}^{\prime}\right\|_{L^{\infty}} \leq C\|\nabla \bar{\varphi}\|_{L^{2}}
$$


where constant $C$ does not depend on $\varepsilon$. Then, by Proposition 4.1, we have the interior $L^{\infty}$ and local Hölder estimate of $\nabla \tilde{\varphi}^{(\varepsilon)}$.

Next for the boundary estimate near $\partial \Omega$, one can apply Theorem 8.29 in [16] to replace Proposition 4.1 to follow the arguments above to show the boundary estimates near $\partial \Omega$.

Step 3. Finally, let us consider the estimates of $\nabla \tilde{\varphi}$ when $|x|$ is sufficiently large. Let

$$
\bar{\psi}^{\prime}:=\partial_{k} \bar{\psi}
$$

where $\bar{\psi}=\bar{\varphi}-q_{\infty} x_{1}$. For any sufficiently large $R$ with $\left\{\frac{R}{2}<|x|<2 R\right\} \subset \Omega$, define

$$
w(y)=R^{\beta^{\prime}} \Phi(R y) \quad \text { and } \quad v(y)=R^{\beta^{\prime}} \bar{\psi}^{\prime}(R y)
$$

on $\left\{\frac{1}{2}<|y|<2\right\}$. Note that $\partial_{i} \bar{\varphi}^{\prime}=\partial_{i} \bar{\psi}^{\prime}$. Then on $\left\{\frac{1}{2}<|y|<2\right\}, w(y)$ satisfies

$$
\sum_{i, j=1}^{n} \frac{\partial}{\partial y_{i}}\left(\tilde{a}_{i j} \frac{\partial}{\partial y_{j}} w\right)=-\sum_{i=1}^{n} \frac{\partial}{\partial y_{i}}\left(\sum_{j=1}^{n} f_{i j} \frac{\partial}{\partial y_{j}} v\right)-\sum_{i=1} \frac{\partial}{\partial y_{i}}\left(R^{\beta^{\prime}+1} \hat{b}_{k} \partial_{i} \varphi^{(\varepsilon)}\right) .
$$

By 2.10 and (3.10), we have that

$$
\int_{\left\{\frac{1}{2}<|y|<2\right\}}|w(y)|^{2} d y \leq C R^{2 \beta^{\prime}-n} \leq C^{\prime} \text {, and } \int_{\left\{\frac{1}{2}<|y|<2\right\}}|v(y)|^{2} d y \leq C R^{2 \beta^{\prime}-n} \leq C^{\prime},
$$

with $R^{\beta^{\prime}+1} \tilde{b}_{k} \partial_{i} \varphi^{(\varepsilon)}$ being bounded by the observation that

$$
\int_{\left\{\frac{1}{2}<|y|<2\right\}}\left|R^{\beta^{\prime}+1} \nabla_{x} \phi(y)\right|^{q} d y \leq C .
$$

The inequality above is due to the second condition for $\phi$ in 2.25 and $\beta^{\prime}+1 \leq \beta+\frac{n}{q}$.

Since $v$ also satisfies $\Delta_{y} v=0$, the standard elliptic estimate implies that

$$
\left\|\frac{\partial}{\partial y_{j}} v\right\|_{L^{\infty}\left(\left\{\frac{1}{2}<|y|<2\right\}\right)} \leq C\|v\|_{L^{2}\left(\left\{\frac{1}{2}<|y|<2\right\}\right)} \leq C,
$$

where constant $C$ only depends on the dimension. Applying Proposition 4.1, we have

$$
|w(y)| \leq C
$$

So let $|y|=1$, then

$$
|\Phi(R y)|=|w(y)| \leq \frac{C}{R^{\beta^{\prime}}} .
$$

Going back to (4.8), we have for sufficiently large $|x|$, and for $i=1, \cdots, n$,

$$
\left|\nabla \tilde{\varphi}^{(\varepsilon)}(x)\right| \leq \frac{C}{|x|^{\beta^{\prime}}}
$$

It means (4.1) holds. Now we can follow the standard argument to lift the regularity to show that $\nabla \tilde{\varphi}^{(\varepsilon)}$ is uniformly Hölder continuous (see [16]), i.e., (4.2) holds. 
LOW MACH NUMBER LIMIT OF MULTIDIMENSIONAL STEADY FLOWS ON THE AIRFOIL PROBLEM

4.2. Uniqueness of solutions of Problem 3. In this subsection, we will show the uniqueness of the modified flow such that we can remove the cut-off and apply the Bers skill to show the existence of solutions of Problem 2.

Lemma 4.2. Problem 3 admits a unique classical solution $\varphi$ up to a constant such that

$$
\int_{\Omega}\left|\nabla \varphi-\left(q_{\infty}, 0, \cdots, 0\right)\right|^{2} d x \leq C
$$

Proof. Exitence is proved by Remark 3.1, Theorem 3.1, and Lemma 4.1. 4.10 follows from (3.10). So we only need to consider the uniqueness.

Assume there are two different solutions of Problem $3 \varphi_{1}$ and $\varphi_{2}$. Let

$$
\hat{\varphi}=\varphi_{1}-\varphi_{2} \quad \text { and } \quad \varphi_{\tau}=(2-\tau) \varphi_{1}+(\tau-1) \varphi_{2} .
$$

Since both $\varphi_{1}$ and $\varphi_{2}$ satisfy 4.10 , we have

$$
\int_{\Omega}|\nabla \hat{\varphi}|^{2} d x \leq C
$$

Moreover, by the straightforward calculation, $\hat{\varphi}$ satisfies that

$$
\sum_{i, j=1}^{n} \partial_{i}\left(\check{a}_{i j} \partial_{j} \hat{\varphi}\right)=0
$$

with

$$
\check{a}_{i j}\left(\nabla \varphi^{(\varepsilon)}\right)=\int_{1}^{2} \hat{\rho}^{(\varepsilon)}\left(\left|\nabla \varphi_{\tau}\right|^{2}, \phi\right)\left(\delta_{i j}-\frac{\hat{q}_{\Lambda}\left(\left|\nabla \varphi_{\tau}\right|^{2}, \phi\right) \partial_{i} \varphi_{\tau} \partial_{j} \varphi_{\tau}}{\left(c^{\varepsilon}\right)^{2}}\right) d \tau .
$$

Define a series of test functions $\eta_{R}(x)>0$ for $R>1$ with uniform $C^{2}$-bounds, such the function $\eta_{R}$ is supported in $|x| \leq R$ and identically equals to 1 in $|x| \leq R-1$.

By multiplying $\hat{\varphi} \eta_{R}$ on both the sides of $(4.12)$ and integrating by part in $\Omega$, we obtain:

$$
\begin{aligned}
0= & \int_{B_{R-1} \cap \Omega} \sum_{i, j=1}^{n} \check{a}_{i j} \partial_{i} \hat{\varphi} \partial_{j} \hat{\varphi} d x . \\
& +\int_{\left(B_{R}-B_{R-1}\right) \cap \Omega} \sum_{i, j=1}^{n} \check{a}_{i j} \partial_{i} \hat{\varphi} \partial_{j} \hat{\varphi} \eta_{R} d x+\int_{\left(B_{R}-B_{R-1}\right) \cap \Omega} \sum_{i, j=1}^{n} \check{a}_{i j} \partial_{i} \hat{\varphi} \partial_{j} \eta_{R} \hat{\varphi} d x
\end{aligned}
$$

By (2.5), 4.1), and (4.11), passing the limit $R \rightarrow \infty$, and by the dominant convergence theorem, the identity above becomes

$$
\int_{\Omega} \sum_{i, j=1}^{n} \check{a}_{i j} \partial_{i} \hat{\varphi} \partial_{j} \hat{\varphi} d x=0
$$

which implies $\nabla \varphi_{1}=\nabla \varphi_{2}$ by $(3.6)$.

Remark 4.1. By Remark 3.1 and Lemma 4.2, it is easy to see when $\left|\nabla \varphi^{(\varepsilon)}\right|<\stackrel{9}{q}_{\theta}^{\varepsilon_{0}}, \varphi^{(\varepsilon)}$ is equal to the solution obtained in [8]. 
4.3. Proof of Theorem 2.3. In this subsection, we will conclude the proof of Theorem 2.3 by showing the solutions of Problem $\mathbf{3}$ are solutions of Problem 2, and then consider the convergence rate of the low Mach number limit.

Proof. Up to now, we have shown that for any given fixed cut-off parameters $\theta$ and $\varepsilon_{0}$, there exists a unique solution of Problem 3, which is denoted as $\varphi^{(\varepsilon)}\left(x ; \varepsilon_{0}, \theta\right)$. It is noticeable that for a given $\theta \in(0,1)$, if $\left|\nabla \varphi^{(\varepsilon)}\left(x ; \varepsilon_{0}, \theta\right)\right|<\stackrel{\circ}{q}_{\theta}^{\varepsilon_{0}}(\phi)$, then $\varphi^{(\varepsilon)}\left(x ; \varepsilon_{0}, \theta\right)$ is the unique solution of Problem 3. Note that

$$
\left|\nabla \varphi^{(\varepsilon)}\left(x ; \varepsilon_{0}, \theta\right)\right|=\left|\varepsilon^{2} \nabla \tilde{\varphi}^{(\varepsilon)}\left(x ; \varepsilon_{0}, \theta\right)+\nabla \bar{\varphi}(x)\right| \leq \max |\nabla \bar{\varphi}|+C\left(\varepsilon_{0}, \theta\right) \varepsilon^{2} .
$$

Then, there exists $\varepsilon_{0, \theta} \leq \varepsilon_{0}$ such that $\left|\nabla \varphi^{(\varepsilon)}\left(x ; \varepsilon_{0}, \theta\right)\right|<\stackrel{\circ}{q}_{\theta}^{\varepsilon_{0}}(\phi)$, for any $0<\varepsilon<\varepsilon_{0, \theta}$. From the definition and uniqueness of $\varphi^{(\varepsilon)},\left\{\varepsilon_{0, \theta}\right\}$ is a non-decreasing sequence respect to $\theta$ with upper bound $\varepsilon_{0}$. Then, we introduce $\varepsilon_{0, c r}=\overline{\lim }_{0<\theta<1} \varepsilon_{0, \theta}$ such that for $0<\varepsilon<$ $\varepsilon_{0, c r}$, there exists a unique solution $\tilde{\varphi}\left(x ; \varepsilon_{0}\right)$, such that

$$
\left|\nabla \varphi^{(\varepsilon)}\left(x ; \varepsilon_{0}\right)\right|=\left|\varepsilon^{2} \nabla \tilde{\varphi}^{(\varepsilon)}\left(x ; \varepsilon_{0}\right)+\nabla \bar{\varphi}(x)\right|<\stackrel{\circ}{q}_{c r}^{\varepsilon_{0}}(\phi),
$$

which means $M^{\varepsilon}(\phi)<1$. In this case, the cut-off can be removed such that the solution is a solution of Problem 2.

After removing the subsonic cut-off, we will optimize the critical value $\varepsilon_{c r}$. For each $0<\varepsilon_{0}<1$, there exists an $\varepsilon_{0, c r}$, with $0<\varepsilon_{0, c r} \leq \varepsilon_{0}<1$. Then, the critical value $\varepsilon_{c}=\sup _{0<\varepsilon<1} \varepsilon_{0, c r}$ satisfies that for any $\varepsilon \in\left(0, \varepsilon_{c}\right), 0<M^{\varepsilon}(\phi)<1$ and $\left|\nabla \tilde{\varphi}^{(\varepsilon)}\right|$ is uniform bounded with respect to $\varepsilon$.

Finally, let us consider the convergence rate of the low Mach number limit. Note that $\varphi^{(\varepsilon)}=\bar{\varphi}+\varepsilon^{2} \tilde{\varphi}^{(\varepsilon)}$ holds in $C^{1, \alpha}(\Omega)$, so $\nabla \varphi^{(\varepsilon)}=\nabla \bar{\varphi}+\nabla \tilde{\varphi}^{(\varepsilon)}$ in the Hölder space, which equals to

$$
u^{\varepsilon}=\bar{u}+\varepsilon^{2} \tilde{u}^{(\varepsilon)} .
$$

It is noticeable that $\phi \in W_{l o c}^{1, q}$ for $q>n$, so $\phi$ is in some Hölder space. Therefore, for the density, by $(2.23), \rho^{\varepsilon} \in C^{\alpha}(\Omega)$. Then $p^{\varepsilon} \in C^{\alpha}(\Omega)$. By the straightforward computation like the one in (4.5), we have

$$
\rho^{\varepsilon}=1+O\left(\varepsilon^{2}\right)
$$

Consequently, the definition of the Mach number yields $M^{\varepsilon}=O(\varepsilon)$. Finally, the gradients of the pressure satisfy

$$
\begin{aligned}
\nabla p^{\varepsilon}-\nabla \bar{p} & =-\operatorname{div}\left(\rho^{\varepsilon} u^{\varepsilon} \otimes u^{\varepsilon}\right)+\operatorname{div}(\bar{u} \otimes \bar{u}) \\
& =\operatorname{div}\left(\bar{u} \otimes \bar{u}-\rho^{\varepsilon} u^{\varepsilon} \otimes u^{\varepsilon}\right) .
\end{aligned}
$$

From 4.17) and 4.18, we can conclude: in the weak sense,

$$
\nabla p^{\varepsilon}=\nabla \bar{p}+O\left(\varepsilon^{2}\right)
$$

It completes the proof of Theorem 2.3 .

Ackowledgments: The authors would like to thank Professor Song Jiang for evaluable suggestions. The research of Mingjie Li is supported by the NSFC Grant No. 11671412. The research of Tian-Yi Wang was supported in part by the NSFC Grant No. 11601401 and the Fundamental Research Funds for the Central Universities(WUT: 2017 IVA 072 and 2017 IVB 066). The research of Wei Xiang was supported in part by the Grants Council of the HKSAR, China (Project No. CityU 21305215, Project No. CityU 11332916, Project No. CityU 11304817 and Project No. CityU 11303518). 


\section{REFERENCES}

[1] T. Alazard, Incompressible limit of the nonisentropic Euler equations with the solid wall boundary conditions. Advances in Differential Equations 10(1) (2005) 19-44.

[2] L. Bers, Existence and uniqueness of a subsonic flow past a given profile. Comm. Pure Appl. Math. 7 (1954) 441-504.

[3] G.-Q. Chen, C. Christoforou, and Y. Zhang, Continuous dependence of entropy solutions to the Euler equations on the adiabatic exponent and Mach number. Arch. Rational Mech. Anal. 189(1) (2008), 97-130.

[4] G.-Q. Chen, F.-M. Huang, T.-Y. Wang, and W. Xiang, Incompressible Limit of Solutions of Multidimensional Steady Compressible Euler Equations, Z. Angew. Math. Phys. (2016) 67-75 .

[5] G.-Q. Chen, F.-M. Huang, T.-Y. Wang, and W. Xiang, Steady Euler flows with Large Vorticity and Characteristic Discontinuities in Arbitrary Infinitely Long Nozzles, Preprint at arXiv:1712.08605 2018.

[6] J. Cheng, L. Du, and W. Xiang, Incompressible Réthy Flows in Two Dimensions, SIAM J. Math. Anal., 49 (2017), 3427-3475.

[7] X. Deng, T.-Y. Wang and W. Xiang, Three-dimensional full Euler flows with nontrivial swirl in axisymmetric nozzles, SIAM J. Math. Anal. 50, 2740-2772.

[8] G.-C. Dong, and B. Ou. Subsonic flows around a body in space, Comm. Partial Differential Equations, 18(1-2) (1993) 355-379.

[9] D. Ebin, The motion of slightly compressible fluids viewed as a motion with strong constraining force. Annals of mathematics, (1977) 141-200.

[10] V. Elling. Nonexistence of low-mach irrotational inviscid flows around polygons. J. Diff. Eqns., 262 (2017) 2705-2721.

[11] B. Fang and W. Xiang, The uniqueness of transonic shocks in supersonic flow past a 2-D wedge, J. Math. Anal. Appl. 437, (2016) 194-213.

[12] R. Finn, and D. Gilbarg, Asymptotic behavior and uniqueness of plane subsonic flows, Comm. Pure Appl. Math. 10 (1957), 23-63.

[13] R. Finn, and D. Gilbarg, Three-dimensional subsonic flows and asymptotic estimates for elliptic partial differential equations, Acta Math. 98 (1957) 265-296.

[14] E. Feireisl, and A. Novotny, Singular Limits in Thermodynamics of Viscous Fluids, Birkhäuser, Basel, 2009.

[15] F. Frankl, and M. Keldysh, Die äussere neumann'she aufgabe für nichtlineare elliptische differentialgleichungen mit anwendung auf die theorie der flugel im kompressiblen gas. Bull. Acad. Sci. 12 (1934) 561-697.

[16] D. Gilbarg, and N. Trudinger, Elliptic partial differential equations of second order, Springer-Verlag, New York,1983, second edition.

[17] X. Gu, and T.-Y. Wang, On subsonic and subsonic-sonic flows in the infinity long nozzle with general conservatives force, Acta Mathematica Scientia 37 (2017) 752-767.

[18] X. Gu, and T.-Y. Wang, On subsonic and subsonic-sonic flows with general conservatives force in exterior domains, Accepted by Acta Mathematicae Applicatae Sinica

[19] H. Isozaki, Singular limits for the compressible Euler equation in an exterior domain, J. Reine Angew. Math., 381 (1987) 1-36.

[20] S. Jiang, Q. Ju and F. Li, Incompressible limit of the non-isentropic ideal magnetohydrodynamic equations, SIAM J. Math. Anal. 48 (2016), no. 1, 302-319.

[21] S. Jiang, Q. Ju, F. Li, and Z.-P. Xin, Low Mach number limit for the full compressible magnetohydrodynamic equations with general initial data. Advances in Mathematics 259 (2014) 384-420.

[22] S. Klainerman and A. Majda, Singular perturbations of quasilinear hyperbolic systems with large parameters and the incompressible limit of compressible fluids, Comm. Pure Appl. Math. 34 (1981) $481-524$.

[23] S. Klainerman and A. Majda, Compressible and incompressible fluids, Comm. Pure Appl. Math. 35 (1982), 629-653.

[24] M. Li, T.-Y. Wang, and W. Xiang, Low Mach Number Limit of Steady Euler Flows in MultiDimensional Nozzles Preprint.

[25] P.-L. Lions and N. Masmoudi, Incompressible limit for a viscous compressible fluid, J. Math. Pures Appl. 77 (1998) 585-627. 
[26] G. Lu, and B. Ou, A Poincare Inequality on $R^{n}$ and Its Application to Potential Fluid Flows in Space. Comm. Appl. Nonlinear Anal. 12(1) (2005) 1-24.

[27] N. Masmoudi, Incompressible, inviscid limit of the compressible Navier-Stokes system, Ann. Inst. H. Poincaré Anal. Non Linéaire, 18 (2) (2001) 199-224.

[28] N. Masmoudi, Examples of singular limits in hydrodynamics, Handbook of differential equations: evolutionary equations, 3, pp. 195-275, 2007.

[29] G. Métivier, and S. Schochet, The incompressible limit of the non-isentropic Euler equations. Arch. for Rational Mech. Anal. 158(1) (2001) 61-90.

[30] B. Ou, An irrotational and incompressible flow around a body in space. J. of PDEs 7(2) (1994) $160-170$.

[31] L. Payne and H. Weinberger, Note on a lemma of Finn and Gilbarg. Acta Math. 98 (1957) 297-299.

[32] A. Qu and W. Xiang, Three-dimensional steady supersonic Euler flow past a concave cornered wedge with lower pressure at the downstream. Arch. for Rational Meth. Anal. 228 (2018) 431-476.

[33] M. Schiffer, Analytical theory of subsonic and supersonic flows in Handbuch der Physik., pp. 1-161, Springer Berlin Heidelberg, 1960.

[34] S. Schochet, The mathematical theory of low Mach number flows. ESAIM: Mathematical Modelling and Numerical Analysis 39(03) (2005) 441-458.

[35] M. Shiffman, On the existence of subsonic flows of a compressible fluid, J. Rational Mech. Anal. 1 (1952) 605-652.

[36] S. Ukai, The incompressible limit and the initial layer of the compressible Euler equation, J. Math. Kyoto Univ. 26 (1986) 323-331.

[37] W. Xiang, Y. Zhang, and Q. Zhao, Two-dimensional steady supersonic exothermically reacting Euler flows with strong contact discontinuity over Lipachitz wall, Preprint at arXiv:1709.03263, 2017.

[38] M. Van Dyke, Perturbation methods in fluid mechanics (Vol. 964), New York: Academic Press, 1964.

M.J. Li, College of Science, Minzu University of China, Beijing 100081, P. R. China

E-mail address: Imjmath@163.com

T.-Y. Wang, Department of Mathematics, School of Science, Wuhan University of Technology, Wuhan, Hubei 430070, P. R. China; Gran Sasso Science Institute, viale Francesco Crispi, 7, 67100 L'Aquila, Italy

E-mail address: tianyiwang@whut.edu.cn; tian-yi.wang@gssi.infn.it; wangtianyi@amss.ac.cn

W. Xiang, City University of Hong Kong, Kowloon Tong, Hong Kong, P. R. China

E-mail address: weixiang@cityu.edu.hk 